\title{
ON CERTAIN RELAXATION OSCILLATIONS: CONFINING REGIONS*
}

\author{
BY \\ PETER J. PONZO AND NELSON WAX \\ University of Illinois
}

\begin{abstract}
Relaxation oscillations described by the generalized Liénard equation, $d^{2} x / d t^{2}+\mu f(x) d x / d t+g(x)=0$, with $\mu \gg 1$, are investigated in the phase and Liénard planes. When $f(x), g(x)$, and $F(x)=\int_{0}^{x} f(u) d u$ are subject to certain restrictions, a number of analytic curves can be obtained in these planes which serve as bounds on solution trajectories. Piece-wise connection of such bounding curves provide explicit annular regions with the property that solution trajectories on the boundary of an annulus move to the interior with increasing time, $t$. The Poincare-Bendixson theorem then guarantees at least one periodic orbit within such an annulus. It is shown that the periodic orbits which are isolated by this means are unique within the annulus, hence orbitally stable. The maximum width of the annulus is of order $\mu^{-4 / 3}$, and the amplitude bounds obtained for the periodic solution agree favorably with the known amplitude for the specific case of the van der Pol equation $d^{2} x / d t^{2}+\mu\left(x^{2}-1\right) d x / d t+x=0$. The results are generalized to less restrictive $f(x), g(x)$, and $F(x)$ than those first considered.
\end{abstract}

1. Introduction. Much attention has been devoted to the periodic solutions of the generalized Liénard equation

$$
\frac{d^{2} x}{d t^{2}}+\mu f(x) \frac{d x}{d t}+g(x)=0, \quad(\mu>0) .
$$

(Cesari [1] contains an extensive bibliography.) Conditions for the existence and uniqueness of non-zero periodic solutions have been investigated; Eq. (1.1) possesses non-zero periodic solutions when $f(x)$ and $g(x)$ are suitably restricted. In particular, a unique non-zero periodic solution of the van der Pol equation

$$
\frac{d^{2} x}{d t^{2}}+\mu\left(x^{2}-1\right) \frac{d x}{d t}+x=0
$$

exists for all $\mu>0$.

Equation (1.1) is equivalent to the systems

$$
\frac{d x}{d t}=\mu v, \frac{d v}{d t}=-\mu f(x) v-g(x) / \mu
$$

and

$$
\frac{d x}{d t}=\mu[y-F(x)], \frac{d y}{d t}=-g(x) / \mu, F(x)=\int_{0}^{x} f(\xi) d \xi,
$$

which define the "scaled" phase $(x, v)$ and Liénard $(x, y)$ planes respectively.

Any initial condition $\left(x\left(t_{0}\right), d x\left(t_{0}\right) / d t\right)$ prescribed for the solution of Eq. (1.1) defines a

*Received July 13, 1964. The research reported in this paper was supported by the Army, Navy and Air Force under Department of Army Contract DA 28043 AMC 00073(E). 
unique point in the phase and Lienard planes. The subsequent development in time of $x(t), d x(t) / d t$ corresponds to a motion of the point in these planes. The curves traced by such motions will be referred to as trajectories, orbits or integral curves, interchangeably. Periodic orbits are simple closed curves in the planes.

In this paper we study a class of relaxation oscillations, namely the periodic solutions of Eq. (1.1) when $\mu$ is very large. We construct explicit annular regions in the scaled Lienard plane with the property that the velocity vectors $(d x / d t, d y / d t)$, associated with points on the boundaries of an annulus, point toward the interior of the annular region. Thus, once a solution trajectory enters the region it must remain therein; by the Poincare-Bendixson theorem, such an annulus contains at least one periodic orbit. However, our annuli contain at most one periodic orbit, which is therefore unique, within the annulus.

It is convenient, at first, to assume that

(1) $f(x)$ and $g(x)$ have continuous first derivatives for all $x$;

(2) there exists an $a<0$ and $a b>0$ such that $f(a)=f(b)=0$ and $f(x)>0$ outside $(a, b) ; F(a)>0, F(b)<0 ; L_{2}(x-a)^{2} \leq F(a)-F(x) \leq L_{1}(x-a)^{2}$ $L_{4}(x-b)^{2} \leq F(x)-F(b) \leq L_{3}(x-b)^{2}$ in $(a, b)$, where $L_{1}, L_{2}, L_{3}, L_{4}>0$ (see Fig. 1);

(3) $x g(x)>0$ for $x \neq 0$;

(4) for $F(x)$, and $G(x)=\int_{0}^{x} g(\xi) d \xi$, that $F( \pm \infty)= \pm \infty, G( \pm \infty)=\infty$;

(5) $g(x) / f(x)$ is monotone decreasing outside $(a, b)$.

Note that $f(x)$ need not be even, nor $g(x)$ odd. Note also that integral curves encircle the origin clockwise as $t$ increases.

Assumptions (1) to (4) are sufficient to assure the existence of a non-zero periodic solution of Eq. (1.1).

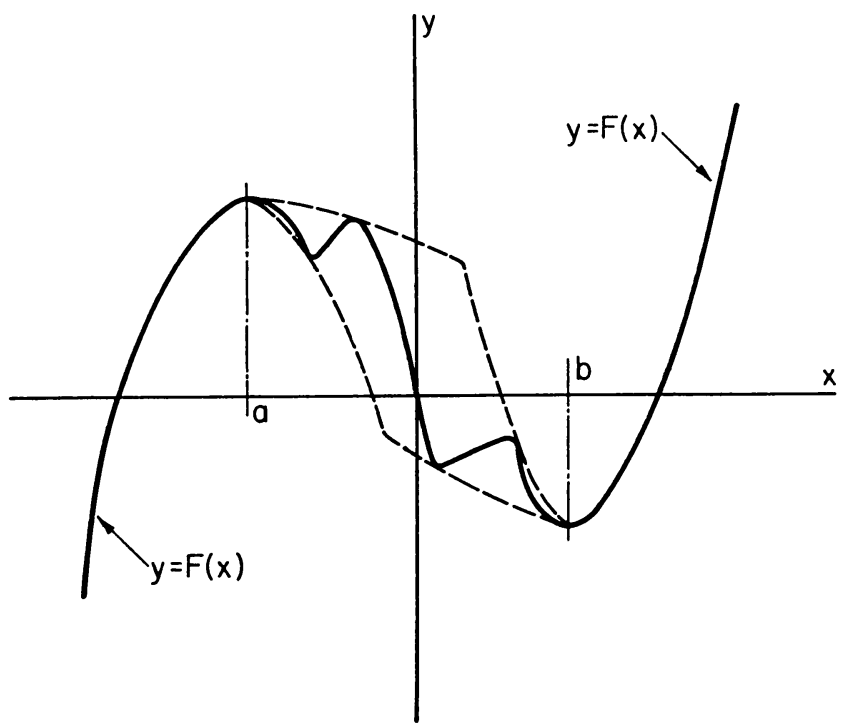

FIg. 1. The region within which $F(x)$ is to lie is shown here. 
Sets of simple confining contours are investigated in Sec. 2. These, and other contours, are used in Sections 3 and 4 to construct inner and outer boundaries of an annulus which contains a periodic solution of Eq. (1.1). The annulus has a maximum width of order $\mu^{-4 / 3}$.

All of the above assumptions, except (3), can be weakened somewhat, as is done in Sec. 5 , where it is shown that the results of previous sections hold, with minor modifications. The boundaries of the annuli given in Sec. 5 still remain within $O\left(\mu^{-4 / 3}\right)$ of each other. LaSalle [2] constructed an annulus of maxumim width $O\left(\mu^{-1}\right)$ for the Liénard equation $(g(x)=x)$, with $f(x)$ subject to different assumptions than those given here.

Section 6 contains some numerical results.

2. Some confining contours. Properties of integral curves have been obtained $[2,3]$ by investigating their relationship to the contours of constant energy

$$
\lambda(x, v)=v^{2} / 2+G(x) / \mu^{2}=c \quad(c=\text { constant }>0)
$$

in the scaled phase plane, or correspondingly the contours

$$
\phi(x, y)=y^{2} / 2+G(x) / \mu^{2}=o
$$

in the scaled Liénard plane. Both the family $\lambda(x, v)=c$ and $\phi(x, y)=c$ are closed nested ovals enclosing the origin: the larger is $c$, the larger the oval.

The time rate of change of $\phi(x, y)$ along a solution $(x(t), y(t))$ of Eq. (1.4) is given by

$$
\frac{d \phi}{d t}(x, y)=y \frac{d y}{d t}+g(x) \frac{d x}{d t} / \mu^{2}=-g(x) F(x) / \mu .
$$

Note that $d \phi / d t$ is the scalar product of the velocity vector $(d x / d t, d y / d t)$ and $\operatorname{grad}(\phi)$, the vector which has the direction of maximum increase in $\phi(x, y)$. Thus, if an integral curve crosses a contour $\phi(x, y)=c$, in a region $R$ where $d \phi / d t \geq 0$, then it does so in a direction of increasing $\phi$, that is, toward the exterior of the oval. Hence, the oval bounds exterior trajectories away from the origin: the contour will be called an "inner bound" in the region $R$. Similarly, if $d \phi / d t \leq 0$ in some region $R^{\prime}$, then $\phi$ ovals are crossed toward their interior and are termed "outer bounds" in $R^{\prime}$.

Let $\alpha<0$ and $\beta>0$ be the two roots of $F(x)=0$ outside $(a, b)$, and assume $G(\alpha) \leq G(\beta)$. Then the oval $\phi_{\alpha}: y^{2} / 2+G(x) / \mu^{2}=G(\alpha) / \mu^{2}$ lies entirely in $\alpha \leq x \leq \beta$. If $F(x)$ were such that $-g(x) F(x) \geq 0$ in $\alpha \leq x \leq \beta$, then $\phi_{\alpha}$ would provide an inner bound on all exterior trajectories. Note that this is the case for the van der Pol equation and, in fact, $\phi_{\alpha}$ is the best " universal" inner bound for equations of the van der Pol type (where $-g F \geq 0$ in $(\alpha, \beta))$, valid for all $\mu>0$ [4].

The $\phi$ ovals are integrals of the Lienard equation when there is no damping $(f(x)=0)$, or, equivalently of the differential equation

$$
d y / d x=\frac{-g(x)}{\mu^{2}[y-F(x)]}
$$

when $F(x)=0$.

Another family of ovals is obtained by letting $F(x)=K$, a constant, in Eq. (2.1). Upon integrating one has

$$
\chi(x, y)=(y-K)^{2} / 2+G(x) / \mu^{2}=\text { constant } .
$$


The $\chi(x, y)=c$ form a family of displaced or shifted, closed, nested ovals. Note that along a solution trajectory $d \chi(x, y) / d t=-g(x)[F(x)-K] / \mu$. Hence the $\chi$ ovals will be inner bounds wherever $d \chi / d t>0$ and outer bounds where $d \chi / d t \leq 0$. In particular, if $x \leq 0$, the $\chi$ ovals are inner bounds where $F(x) \geq \mathrm{K}$ and outer bounds where $F(x) \leq K$.

We introduce now a new set of contours in the phase plane. From Eq. (1.3) one has that

$$
d v / d x=-f(x)-g(x) / \mu^{2} v
$$

which can be written as

$$
v d v+g(x) d x / \mu^{2}+f(x) v d x=0 .
$$

On integrating, this becomes

$$
v^{2} / 2+G(x) / \mu^{2}+\int v d F(x)=\text { constant } .
$$

Integrating by parts, one gets

$$
v^{2} / 2+G(x) / \mu^{2}+v F(x)-\int F(x) d v=\text { constant. }
$$

On setting $F(x)=K$, a constant, in the integral, one obtains the family of contours

$$
v^{2} / 2+G(x) / \mu^{2}+v[F(x)-K]=\text { constant. }
$$

We consider a particular set of these contours, namely the one parameter family

$$
\Psi(x, v)=v^{2} / 2+v[F(x)-F(u)]+G(x) / \mu^{2}=G(u) / \mu^{2} .
$$

The upper branch of such a contour satisfies

$$
v=F(u)-F(x)+\left\{[F(u)-F(x)]^{2}+2[G(u)-G(x)] / \mu^{2}\right\}^{1 / 2} \geq 0
$$

for $u \leq x \leq 0$, in the second quadrant (see Fig. 2).

We determine the region in which these contours are inner bounds by a direct comparison of slopes. One has, from Eq. (2.4), that

$$
d v / d x=-\frac{f(x) v+g(x) / \mu^{2}}{v+F(x)-F(u)}=\frac{1}{1+v^{-1}[F(x)-F(u)]}\left[-f(x)-g(x) / \mu^{2} v\right] .
$$

Since $\left[-f(x)-g(x) / \mu^{2} v\right]$ is just the slope of trajectories, and is positive for $0<v<$ $v_{0}(x)=-g(x) / \mu^{2} f(x)$, in $x<a$, then the upper branch (Eq. (2.5)) of a $\Psi$ ) contour will be an inner bound wherever $F(x) \geq F(u)$, as long as the $\Psi$ contour remains below $v_{0}(x)$. We will show below, however, that $v=v_{0}(x)$ is an outer bound in $x<a$. Consider then the possibility of an intersection, from below, of a $\Psi$ contour with $v=v_{0}(x)$. All trajectories between the $\Psi$ contour and $v_{0}(x)$ must converge to such an intersection, which would make it a singular point, contradicting the uniqueness guaranteed by our 


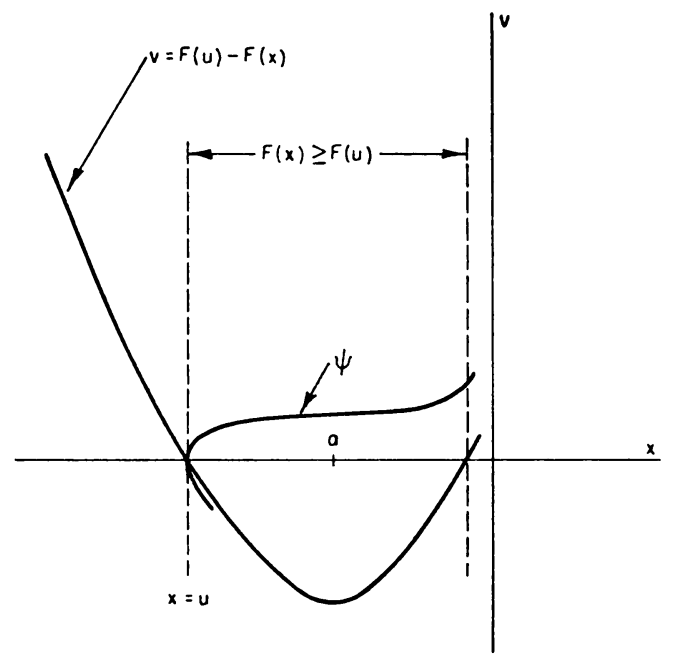

Fig. 2. An excluding $\psi$ contour is shown in the phase plane.

assumptions. We conclude that the $\Psi$ contours provide inner bounds for $F(x) \geq F(u)$.

In the scaled Liénard plane the $\Psi$ contours become

$$
[y-F(u)]^{2} / 2-[F(x)-F(u)]^{2} / 2+G(x) / \mu^{2}=G(u) / \mu^{2}
$$

or, choosing the upper branch,

$$
y=F(u)+\left\{[F(x)-F(u)]^{2}+2[G(u)-G(x)] / \mu^{2}\right\}^{1 / 2} .
$$

Consider now the curve $v_{0}(x)=-g(x) / \mu^{2} f(x)$, which, from Eq. (2.3), is the contour of zero slope in the phase plane. We have assumed that $g(x) / f(x)$ is monotone decreasing outside $(a, b)$, so that trajectories which start on, or cross $v_{0}(x)$, in $x<a$, will move to the right away from and below $v_{0}(x)$. Thus $v=v_{0}(x)$ is an outer bound in $x<a$, and similarly in $x>b$ (see Fig. 3). Notice that any monotone increasing curve lying on or above $v_{0}(x)$, in the phase plane $(x<a)$, will serve as an outer bound.

Other bounding arcs will be established on the next sections.

3. The outer boundary. Consider a Liénard trajectory which starts on, or below,

$$
\Gamma_{0}: y=F(x)-g(x) / \mu^{2} f(x),
$$

the Liénard plane representation of $V_{0}(x)$, for $x<a$. $\Gamma_{0}$ furnishes an outer bound; at $x=a$, however, $\Gamma_{0}$ is singular. An improvement on $\Gamma_{0}$ in the neighborhood of $x=a$ can be found by using a $\chi$ oval.

The $\chi$ ovals

$$
\chi(x, y)=(y-K)^{2} / 2+G(x) / \mu^{2}=\text { constant }
$$

are outer bounds, for $x \leq 0$,

$$
\chi^{\cdot}=-g(x)[F(x)-K] / \mu \leq 0,
$$




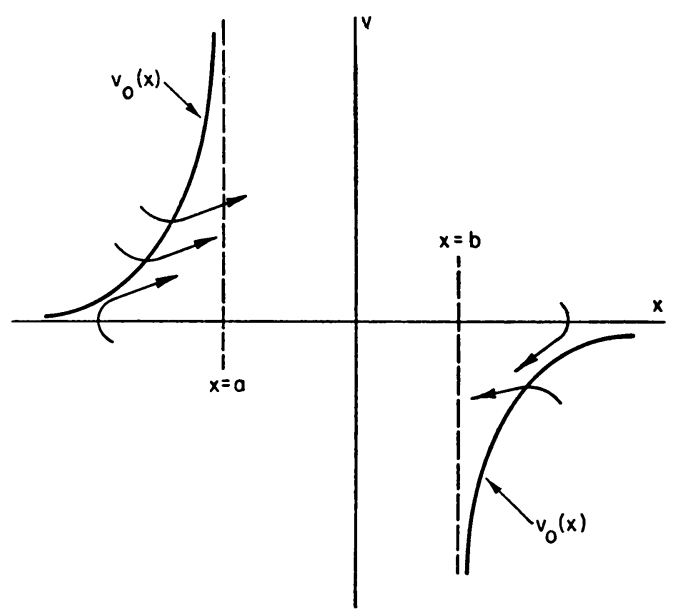

Fig. 3. The zero-slope isocline, $v_{0}(x)$, is shown in the phase plane.

that is, wherever $F(x) \leq K$. Thus, if one chooses $K=\max [F(x)]=F(a)$ (in $x \leq 0$ ), then all integral curves will cross these ovals from the exterior to the interior, when $x \leq 0$. The constant can be given by $G(k) / \mu^{2}$ and these $\chi$ ovals represented by

$$
\chi(x, y)=[y-F(a)]^{2} / 2+G(x) / \mu^{2}=G(k) / \mu^{2} .
$$

A member of this family will join smoothly* onto $\Gamma_{0}$ if $k$ is chosen properly.

The equations

$$
Y_{1}=F\left(X_{1}\right)-g\left(X_{1}\right) / \mu^{2} f\left(X_{1}\right)=F(a)+\left\{2\left[G(k)-G\left(X_{1}\right)\right]\right\}^{1 / 2} / \mu
$$

and

$$
[d y / d x]_{X_{1}, Y_{1}}=\frac{-g\left(X_{1}\right)}{\mu\left\{2\left[G(k)-G\left(X_{1}\right)\right]\right\}^{1 / 2}}=f\left(X_{1}\right)-\frac{1}{\mu^{2}} \frac{d}{d x}[g(x) / f(x)]_{X_{1}, Y_{1}}
$$

determine the appropriate choice of $k, k_{1}$, and the coordinates $\left(X_{1}, Y_{1}\right)$ of $P_{1}$ (the point of tangency); $P_{1}$ and the $\chi$ oval, $\chi_{1}$, are thereby fixed.

The $\chi_{1}$ oval could be used as an outer bound to the $y$-axis, but it is too crude. Instead, we use only the $\operatorname{arc} P_{1} P_{2}$ of $\chi_{1}$ to $P_{2}$ (to be determined) and continue from $P_{2}$ with another contour.

The integral curve through $P_{2}$ satisfies

$$
0 \leq d y / d x=\frac{-g(x)}{\mu^{2}[y-F(x)]} \leq \frac{-g(x)}{\mu^{2}\left[Y_{2}-F(x)\right]}
$$

for $X_{2} \leq x \leq 0$, where $\left(X_{2}, Y_{2}\right)$ are the coordinates of $P_{2}$. Now

$$
y-F(x) \geq y-F(a)+L_{2}(x-a)^{2} \geq Y_{2}-F(a)+L_{2}(x-a)^{2},
$$

*Here and elsewhere, "smoothly" is to imply a continuous tangent. 
from the assumed properties of $F(x)$.

Let

$$
M_{2}=\max [-g(x)], \quad a \leq x \leq 0,
$$

and

$$
\gamma_{2}^{2}=\left[Y_{1}-F(a)\right] / L_{2}>0,
$$

since $Y_{2}>Y_{1}>F(a)$. Then

$$
d y / d x \leq d y_{u} / d x=\frac{1}{\mu^{2}} \frac{M_{2} / L_{2}}{\gamma_{2}^{2}+(x-a)^{2}} .
$$

The integral of this last equation which passes through $P_{2}$,

$$
y_{u}(x)=Y_{2}+\frac{M_{2}}{\mu^{2} \gamma_{2} L_{2}}\left[\tan ^{-1}\left(\frac{x-a}{\gamma_{2}}\right)-\tan ^{-1}\left(\frac{X_{2}-a}{\gamma_{2}}\right)\right],
$$

serves as an outer bound from $P_{2}$ to $P_{3}\left(0, Y_{3}\right)$.

We now determine the point $P_{2}$ by requiring that $y_{u}(x)$ join smoothly onto $\chi_{1}$ at $P_{2}$. The equality of slopes

$$
\frac{1}{\mu^{2}} \frac{M_{2} / L_{2}}{\gamma_{2}^{2}+\left(X_{2}-a\right)^{2}}=\frac{-g\left(X_{2}\right)}{\mu\left\{2\left[G\left(k_{1}\right)-G\left(X_{2}\right)\right]\right\}^{1 / 2}}
$$

determines $X_{2}$. The $\chi_{1}$ oval and $X_{2}$ being known, $P_{2}$ is now fixed.

The point $P_{3}\left(0, Y_{3}\right)$ is obtained from Eq. (3.3)

$$
Y_{3}=y_{u}(0)=Y_{2}+\frac{M_{2}}{\mu^{2} \gamma_{2} L_{2}}\left[\tan ^{-1}\left(-a / \gamma_{2}\right)-\tan ^{-1}\left(\frac{X_{2}-a}{\gamma_{2}}\right)\right] \text {. }
$$

The outer bound from $P_{3}$ to $P^{1}$ is an arc of another $\chi$ oval, $\chi_{3}$. From $d \chi / d t=-g(x)$ $[F(x)-K] / \mu$ we have that $d \chi / d t \leq 0$, for $x \geq 0$, wherever $F(x) \geq K$. Choosing $K=$ $F(b)$ (the minimum value of $F(x)$ for $x \geq 0$ ), then $d x / d t \leq 0$ and the oval through $P_{3}$ is selected to be the outer bound. Thus,

$$
y=F(b)+\left\{\left[Y_{3}-F(b)\right]^{2}-2 G(x) / \mu^{2}\right\}^{1 / 2}
$$

is the outer bound from $P_{3}$ to $P_{4}$, where this oval intersects $y=F(x)$. Note that this outer bound is a horizontal line segment, $y=Y_{3}$, to within $O\left(\mu^{-2}\right)$. The point $P_{4}$ has as coordinates: $X_{4}$, the solution of

$$
F(x)=F(b)+\left\{\left[Y_{3}-F(b)\right]^{2}-2 G(x) / \mu^{2}\right\}^{1 / 2},
$$

and $Y_{4}=F\left(X_{4}\right)$.

The outer bound from $P_{4}$ to $P_{5}$, a point on $\Gamma_{0}$, is chosen to be a short (length $O\left(\mu^{-2}\right)$ ) vertical line segment, connecting $P_{4}$ with the point $P_{5}$ directly below it on $\Gamma_{0}$. Note that trajectories cross this vertical segment from right to left $(d x / d t<0$ for $y<F(x))$ and it is thus an outer bound.

The outer bound now follows $\Gamma_{0}$ from $P_{5}$ to $P_{6}$, which corresponds to $P_{1}$. The point $P_{7}$ is chosen just as $P_{2}$ was, etc. 
The outer boundary of the annulus is now complete (Fig. 4).

Approximate expressions for the various coordinates and contours can be found by expanding the equations in Taylor's series and retaining terms in the appropriate orders.

To determine $P_{1}$ we substitute $X_{1}=a-\Delta_{1} \mu^{-2 / 3}$ and $k_{1}=a-\xi \mu^{-2 / 3}$ in Eq's. (3.2) and calculate that

$$
\begin{aligned}
\Delta_{1} & =\left\{|g(a)| /\left[f^{\prime}(a)\right]^{2}\right\}^{1 / 3}+O\left(\mu^{-2 / 3}\right) \\
\xi & =9 \Delta_{1} / 8+O\left(\mu^{-2 / 3}\right) .
\end{aligned}
$$

We then find

$$
Y_{1}=F(a)+\frac{1}{2}\left\{g^{2}(a) /\left|f^{\prime}(a)\right|\right\}^{1 / 3} \mu^{-4 / 3}+O\left(\mu^{-2}\right) .
$$

For $P_{2}$ we set $X_{2}=a+\Delta_{2} \mu^{-2 / 3}$ in Eq. (3.4) and obtain a quartic for the determination of $\Delta_{2}$ :

$$
\left(\Delta_{2}^{2}+W_{2}^{2}\right)^{2}=\frac{2 M_{2}^{2}}{|g(a)| L_{2}^{2}}\left(\Delta_{2}+\xi\right)
$$

where

$$
W_{2}^{2}=\mu^{4 / 3}\left[Y_{1}-F(a)\right] / L_{2}=\frac{1}{2}\left\{g^{2}(a) /\left|f^{\prime}(a)\right|\right\}^{1 / 3} / L_{2}+O\left(\mu^{-2 / 3}\right) .
$$

From the equation for the $\chi_{1}$ oval, we get

$$
Y_{2}=F(a)+\left\{2|g(a)|\left(\Delta_{2}+\xi\right)\right\}^{1 / 2} \mu^{-4 / 3}+O\left(\mu^{-2}\right) .
$$

From Eq. (3.5) we have, for $P_{3}$,

$$
Y_{3}=Y_{2}+\frac{M_{2}}{W_{2} L_{2}}\left\{\pi / 2-\tan ^{-1}\left(\Delta_{2} / W_{2}\right)\right\} \mu^{-4 / 3}+O\left(\mu^{-2}\right) .
$$

Since the $\operatorname{arc} P_{3} P_{4}$ is horizontal, to $O\left(\mu^{-2}\right)$, then $Y_{4}=Y_{3}+O\left(\mu^{-2}\right)=F\left(X_{4}\right)$. From

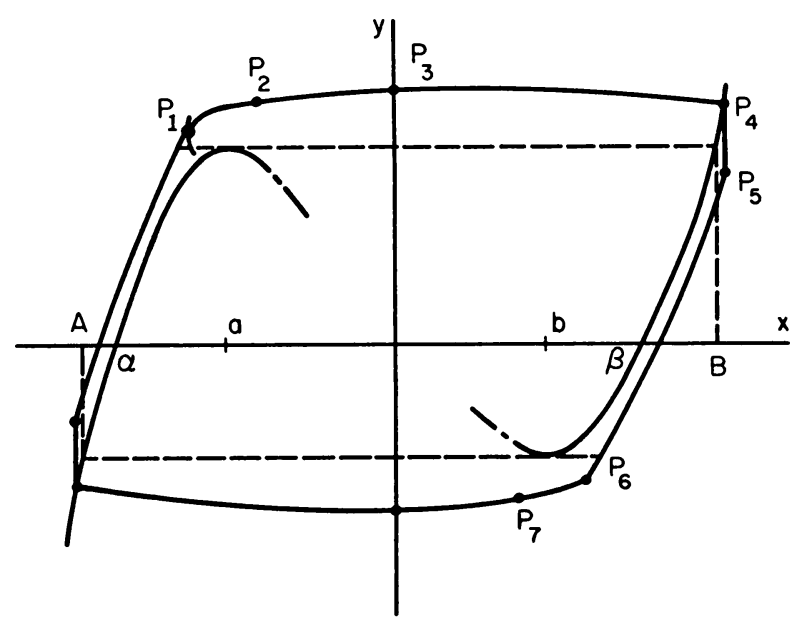

Fig. 4. The outer boundary of the confining annulus is shown in the Lienard plane. 
this relation, we find $X_{4}$ by setting $X_{4}=B+\epsilon$, where $B$ is the smallest positive root of $F(x)=F(a)$. This gives

Then, finally,

$$
\epsilon=\left[Y_{4}-F(a)\right] / f(B)+O\left(\mu^{-2}\right) .
$$

$X_{4}=B+\frac{1}{f(B)}\left[\left\{2|g(a)|\left(\Delta_{2}+\xi\right)\right\}^{1 / 2}+\frac{M_{2}}{W_{2} L_{2}}\left\{\pi / 2-\tan ^{-1}\left(\Delta_{2} / W_{2}\right)\right\}\right]^{-4 / 3}+O\left(\mu^{-2}\right)$.

Note that $X_{4}$ gives an upper bound on the maximum positive $x$-excursion of the periodic orbit. In Section 6 we will apply this bound to a particular equation. If we define $A$ as the maximum negative root of $F(x)=F(b)$, then an analogous bound, involving $A$, may be obtained for the maximum negative $x$-excursion of the periodic orbit.

4. The inner boundary. Consider an integral curve which starts at $p_{1}\left(x_{1}, y_{1}\right)$, $x_{1} \leq \alpha, y_{1}=F\left(x_{1}\right)$. The $\Psi$ contour through $p_{1}$

$$
\Psi_{1}=\left[y-F\left(x_{1}\right)\right]^{2} / 2-\left[F(x)-F\left(x_{1}\right)\right]^{2} / 2+G(x) / \mu^{2}=G\left(x_{1}\right) / \mu^{2}
$$

provides an inner bound on the trajectory through $p_{1}$, since $F(x)>F\left(x_{1}\right)$ in $x_{1} \leq x \leq a$. Furthermore, this trajectory is exterior to all $\Psi$ contours for which $x_{1} \leq u \leq a$. Hence the trajectory through $p_{1}$ will rise above the envelope to the family of $\Psi$ contours (see Fig. 5).

Thus, an inner bound on the trajectory through $p_{1}$ is the arc of the $\Psi_{1}$ oval to $p_{2}$, where the oval joins smoothly onto the envelope $E$. The envelope will provide a continuation of the inner bound from $p_{2}$. Parametric equations for $E$ are

$$
\begin{aligned}
E: y-F(x) & =-g(u) / \mu^{2} f(u) \\
G(x) & =G(u)-\frac{1}{2 \mu^{2}}[g(u) / f(u)]^{2}+[F(x)-F(u)] g(u) / f(u) .
\end{aligned}
$$

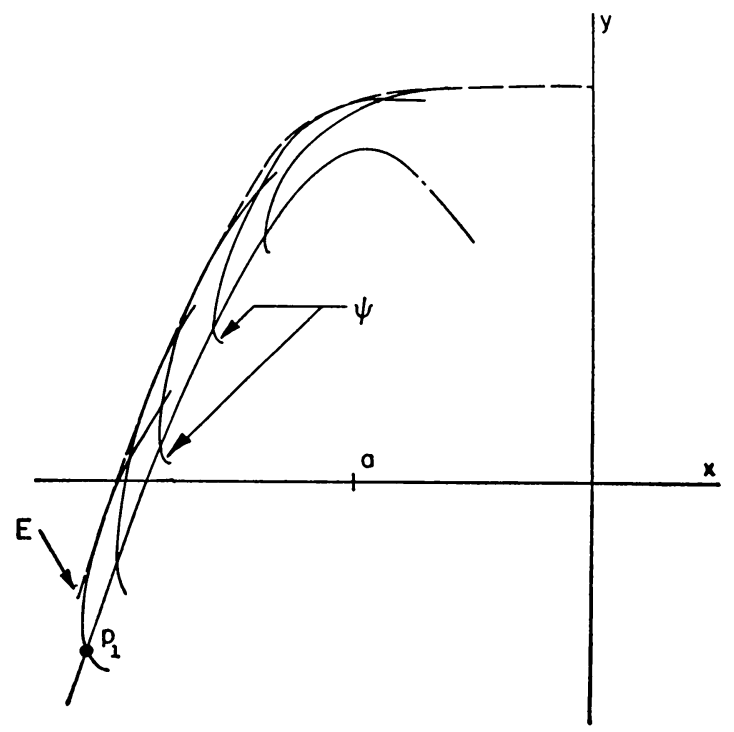

Fia. 5. The envelope, $E$, of the family of $\psi$ contours is depicted, in the Liénard plane. 
When $u$ is given, Eq's. (4.2) determine a point, $p_{u}$, on the envelope $E$; the locus of such points is $E$. However, $E$ will be an inner bound only at points for which $d \Psi / d t \geq 0$, namely when $F(x) \geq F(u)$ for $x<0$. Consequently, associated with each $u$ is a $\Psi$ oval (Eq. (2.6)), an excluding interval where $F(x) \geq F(u)$, and a point, $p_{u}$, on $E$. If $p_{u}$ lies within the excluding interval then $E$ will be an inner bound at this point, otherwise not. Figure 6 illustrates the geometrical significance of Eqs. (4.2) where, for clarity, we have used the scaled phase plane representation of $E$, that is

$$
\begin{aligned}
v & =-g(u) / \mu^{2} f(u)=v_{0}(u) \\
G(x) & =G(u)-\frac{1}{2 \mu^{2}}[g(u) / f(u)]^{2}+[F(x)-F(u)] g(u) / f(u) .
\end{aligned}
$$

Let $u_{m}$ be the maximum value of $u$ for which $p_{u_{m}}\left(=p_{3}\right)$ lies within the excluding interval; $E$ will be an inner bound for $u \leq u_{m}$. The coordinates of $p_{3}\left(x_{3}, y_{3}\right)$ are obtained by solving Eqs. (4.2) together with $F\left(x_{3}\right)=F\left(u_{m}\right)$. Clearly $u_{m}<a$ and $x_{3}>a$ (Fig. 7).

The equations which determine $x_{3}, y_{3}$ and $u_{m}$ are

$$
\begin{aligned}
y & =F(x)-g(u) / \mu^{2} f(u) \\
G(x) & =G(u)-\frac{1}{2 \mu^{2}}[g(u) / f(u)]^{2}+[F(x)-F(u)] g(u) / f(u) \\
F(x) & =F(u) .
\end{aligned}
$$

The point $p_{3}$ (in the scaled Liénard plane) at which $E$ stops being an inner bound, lies to the right, and above, the point $(a, F(a))$ where $y=F(x)$ is a maximum.

It is important to observe that $p_{3}$ is determined directly from the properties of $f(x), g(x)$ and the value of $\mu$, and not from $p_{1}$ and $p_{2}$.

The inner bound from $p_{3}$ to $p_{4}\left(0, y_{4}\right)$ is obtained by bounding the slopes of the trajectories in the interval $x_{3} \leq x \leq 0$. Since $d y / d x \geq 0$ in this interval (when $y>F(x)$ ),

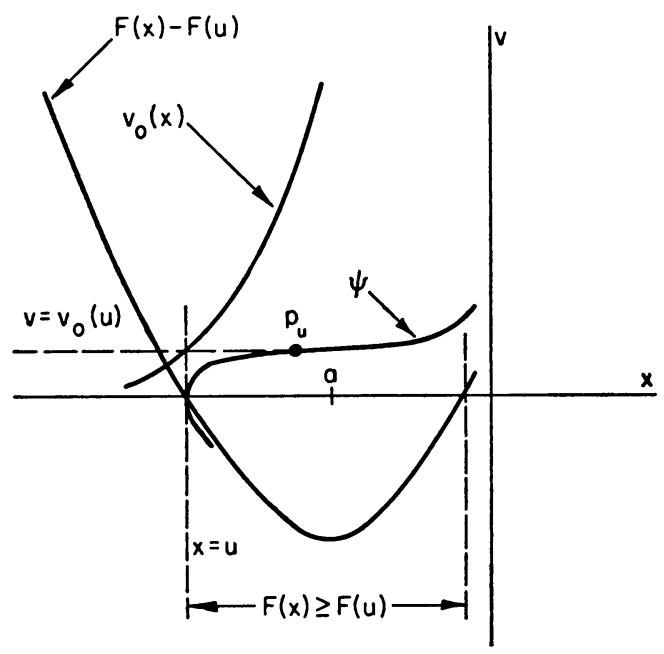

Fig. 6. A geometrical interpretation of the parametric equations for the envelope $E$, is shown, in the phase plane. 


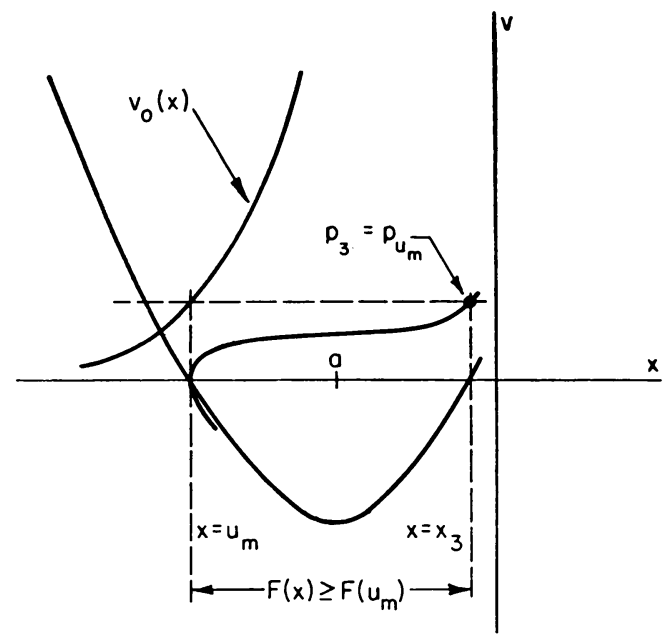

FIG. 7. The largest parameter value, $u_{m}$, for which the envelope is an inner bound, is illustrated in the phase plane.

it follows that a trajectory starting at $y\left(x_{3}\right)$ rises monotonically to $y(0)$. Since $y(0) \leq Y_{3}$, the outer bound at $x=0$ (Eq. (3.5)), it follows that

$$
y-F(x) \leq y-F(a)+L_{1}(x-a)^{2} \leq Y_{3}-F(a)+L_{1}(x-a)^{2}
$$

from the assumed properties of $F(x)$. Furthermore, a constant $C$ can be found such that $-g(x) \geq-g(a)+C(x-a)$. Thus

$$
d y / d x \geq d y_{L} / d x=\frac{1}{\mu^{2}} \frac{-g(a)+C(x-a)}{Y_{3}-F(a)+L_{1}(x-a)^{2}} .
$$

The integral of the last equation which passes through $p_{3}$ is

$$
\begin{aligned}
y_{L}(x)=y_{3}-\frac{g(a)}{\gamma_{1} L_{1} \mu^{2}}\left[\tan ^{-1}\left(\frac{x-a}{\gamma_{1}}\right)-\tan ^{-1}\left(\frac{x_{3}-a}{\gamma_{1}}\right)\right] \\
+\frac{C}{2 L_{1} \mu^{2}} \log \left[\frac{\gamma_{1}^{2}+(x-a)^{2}}{\gamma_{1}^{2}+\left(x_{3}-a\right)^{2}}\right],
\end{aligned}
$$

where $\gamma_{1}^{2}=\left[Y_{3}-F(a)\right] / L_{1} ; y_{L}(x)$ can serve as a lower bound on the trajectory from $p_{3}$ to $p_{4}$. The point $p_{4}$ is determined from Eq. (4.4), at $x=0$, as

$$
\begin{aligned}
y_{4}=y_{L}(0)=y_{3}-\frac{g(a)}{\gamma_{1} L_{1} \mu^{2}}\left[\tan ^{-1}(-a / \gamma)-\tan ^{-1}\left(\frac{x_{3}-a}{\gamma_{1}}\right)\right] \\
+\frac{C}{2 L_{1} \mu^{2}} \log \left[\frac{\gamma_{1}^{2}+a^{2}}{\gamma_{1}^{2}+\left(x_{3}-a\right)^{2}}\right] .
\end{aligned}
$$

The $\operatorname{arc} p_{4} p_{5}$ is also obtained by integrating a slope inequality. In $x \geq 0, y \geq F(x)$, the slope of trajectories satisfies

$$
0 \geq d y / d x=\frac{-g(x)}{\mu^{2}[y-F(x)]} \geq \frac{-g(x)}{\mu^{2}\left[y_{0}-F(x)\right]}
$$


as long as $y(x)>y_{0}$, where $y_{0}$ is a constant to be chosen. Now $y_{0}-F(x)$ has a simple zero at $x=x_{0}>0$, where $y_{0}=F\left(x_{0}\right)$; hence one may write $\left[y_{0}-F(x)\right] /\left(x_{0}-x\right)=H(x)>0$ for $x \geq 0$. We will choose $y_{0} \geq F(a)$, so that $x_{0} \geq B$, where $B$ is the unique positive root of $F(x)=F(a)$. Hence $H(x) \geq h(x)=[F(B)-F(x)] /(B-x)>0$, for $x \geq 0$. On setting $M_{1}=\max [g(x) / h(x)], 0 \leq x \leq X_{4}$, where $X_{4}$ is the maximum positive $x$-excursion of the outer bound (Eq. (3.7)), one has

$$
d y / d x \geq \frac{-M_{1}}{\mu^{2}\left(x_{0}-x\right)}
$$

We may then choose as inner bound the integral of $d y / d x=-M_{1} / \mu^{2}\left(x_{0}-x\right)$ which passes through $p_{4}$, namely

$$
y(x)=y_{4}+\frac{M_{1}}{\mu^{2}} \log \left(\frac{x_{0}-x}{x_{0}}\right) .
$$

This inner bound is valid until $y=y_{0}$ (see Fig. 8). We choose $y_{0}$ such that this inner bound intersects $y=y_{0}$ at $x=x_{0}\left(1-1 / \mu^{2}\right)$. Then $y_{0}=y_{4}-2 M_{1} \log \mu / \mu^{2}$. Our inner bound is thus

$$
y=y_{4}+\frac{M_{1}}{\mu^{2}} \log \left(\frac{x_{0}-x}{x_{0}}\right)
$$

from $p_{4}\left(0, y_{4}\right)$ to $p_{5}$ whose coordinates are $x_{5}=x_{0}\left(1-1 / \mu^{2}\right), y_{5}=y_{4}-2 M_{1} \log \mu / \mu^{2}$, with $y_{5}=F\left(x_{0}\right)$.

The inner bound from $p_{5}$ to $p_{6}$ (the point of intersection with $y=F(x)$ ) is chosen to be an arc of the $\Psi$ contour, $\Psi_{5}$, through $p_{5}$. This contour is determined by finding the value of the parameter, $u_{5}$, from

$$
y_{5}=F\left(u_{5}\right)+\left\{\left[F\left(x_{5}\right)-F\left(u_{5}\right)\right]^{2}+2\left[G\left(u_{5}\right)-G\left(x_{5}\right)\right] / \mu^{2}\right\}^{1 / 2} .
$$

Then $\Psi_{5}$ will intersect $F(x)$ at $p_{6}\left(x_{6}, y_{6}\right)$ where $x_{6}=u_{5}, y_{6}=F\left(u_{5}\right)$. This contour will also join the envelope $E$, at $p_{7}$.

The remainder of the inner boundary of the annulus is constructed similarly, with $p_{6}$ corresponding to $p_{1}$ and $p_{7}$ to $p_{2}$.

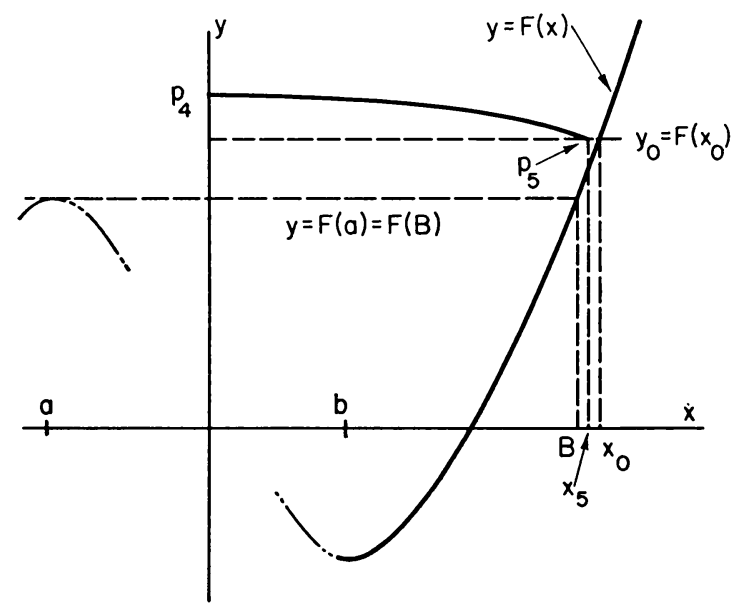

Fig. 8. The Liénard plane construction of the inner bound from $p_{4}$ to $p_{5}$ is given. 
The inner boundary is now complete. (Fig. 9).

Approximate expressions for the coordinates and contours are obtained as in Sec. 3.

We will first eliminate the parameter $u$ from Eq's. (4.2) and obtain an equation for the envelope $E$, valid for all $u \leq a-\epsilon(\epsilon>0$ arbitrary). From the second equation we determine that $x=u+0\left(\mu^{-1}\right)$; thus, in the first equation we find

$E$ :

$$
y=F(x)-g(x) / \mu^{2} f(x)+O\left(\mu^{-3}\right), \quad(x \leq a-\epsilon) .
$$

Observe that the envelope $E$ lies only $O\left(\mu^{-3}\right)$ from $\Gamma_{0}$ in this region $(x \leq a-\epsilon)$; that is, the annulus has width $O\left(\mu^{-3}\right)$ here, and similarly for $x \geq b+\epsilon$.

To determine $p_{3}$ we set $x_{3}=a+\delta_{3} \mu^{-2 / 3}$ and $u_{m}=a-\eta \mu^{-2 / 3}$ in Eq. (4.3) and calculate

$$
\begin{aligned}
\delta_{3} & =\left\{|g(a)| /\left[2 f^{\prime}(a)\right]^{2}\right\}^{1 / 3}+O\left(\mu^{-2 / 3}\right) \\
\eta & =\delta_{3}+O\left(\mu^{-2 / 3}\right)
\end{aligned}
$$

and, consequently, we find

$$
y_{3}=F(a)+\frac{7}{8}\left\{4 g^{2}(a) /\left|f^{\prime}(a)\right|\right\}^{1 / 3} \mu^{-4 / 3}+O\left(\mu^{-2}\right) .
$$

For $p_{4}$ we get, from Eq. (4.5)

$$
y_{4}=y_{3}+\frac{|g(a)|}{W_{1} L_{1}}\left\{\pi / 2-\tan ^{-1}\left(\delta_{3} / W_{1}\right)\right\} \mu^{-4 / 3}+O\left(\log \mu / \mu^{2}\right),
$$

where $W_{1}^{2}=\mu^{4 / 3}\left[Y_{3}-F(a)\right] / L_{1}$ ( $Y_{3}$ is obtained from the outer bound).

Since the $\operatorname{arc} p_{4} p_{5} p_{6}$ is horizontal, to within $O\left(\log \mu / \mu^{2}\right)$, then $y_{6}=y_{4}+0\left(\log \mu / \mu^{2}\right)$ $=F\left(x_{6}\right)$, which determines $x_{6}$. We find, as for the outer bound in Sec. 3 , that

$$
\begin{aligned}
x_{6}=B+\frac{1}{f(B)}\left[\frac{7}{8}\left\{4 g^{2}(a) /\left|f^{\prime}(a)\right|\right\}^{1 / 3}\right. & \\
& \left.+\frac{|g(a)|}{W_{1} L_{1}}\left\{\pi / 2-\tan ^{-1}\left(\delta_{3} / W_{1}\right)\right\}\right] \mu^{-4 / 3}+O\left(\log \mu / \mu^{2}\right) .
\end{aligned}
$$

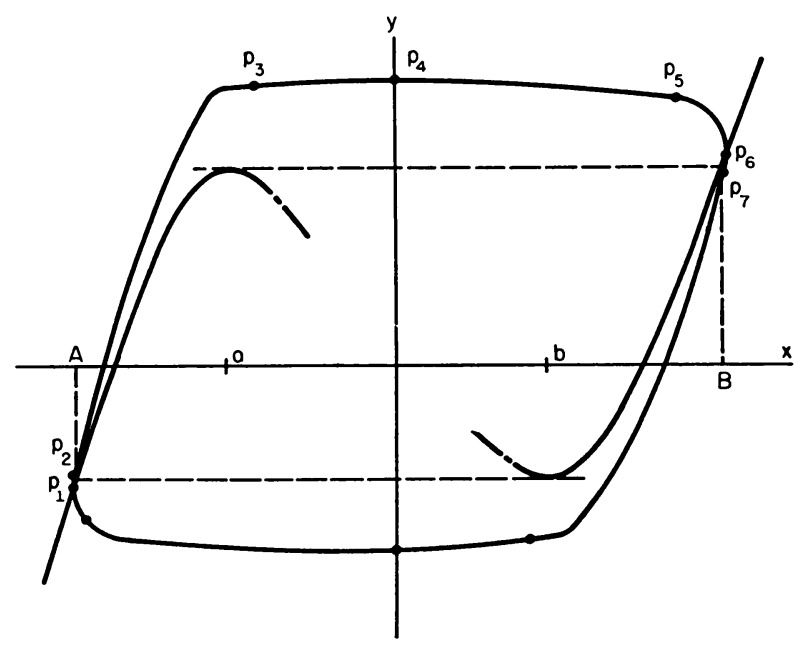

Fig. 9. The inner boundary of the confining annulus is presented, in the Liénard plane. 
Observe that $x_{6}$ provides a lower bound on the maximum positive $x$-excursion of the periodic orbit.

5. Generalizations. The assumptions of Section 1 are needlessly restrictive. It is possible to weaken them somewhat and still obtain an annulus of maximum width $O\left(\mu^{-4 / 3}\right)$, as is done here.

Let $a, b, A, B$ have their previous meanings, and let $\epsilon>0$ be an arbitrary positive number.

Assume that

(1) $g(x)$ satisfies a Lipschitz condition in $A-\epsilon \leq x \leq B+\epsilon$ with $x g(x)>0$ for $x \neq 0$ in this interval;

(2) a) $f(x)$ is continuous in $A-\epsilon \leq x \leq B+\epsilon$;

b) $f(x)>0$ in $A-\epsilon \leq x<a$, and $b<x \leq B+\epsilon$;

c) there exist constants $K_{1}>0$, and $K_{2}>0$ such that

$$
\begin{array}{ll}
f(x)>K_{1}(a-x) & A-\epsilon \leq x<a \\
f(x)>K_{2}(x-b) & b<x \leq B+\epsilon ;
\end{array}
$$

(3) $F(a)>0, F(b)<0$, and that there exist positive constants $L_{1}, L_{2}, L_{3}$, $L_{4}$ such that

$$
\begin{aligned}
& L_{2}(a-x)^{2} \leq F(a)-F(x) \leq L_{1}(a-x)^{2} \\
& L_{4}(x-b)^{2} \leq F(x)-F(b) \leq L_{3}(x-b)^{2}
\end{aligned}
$$

in $a \leq x \leq b$.

Cartwright [5] has shown that $f(x)$ continuous, $g(x)$ Lipschitz, are sufficient for the existence and uniqueness of solutions of the phase plane equation. In the Lienard plane, $y-F(x)$ is continuously differentiable.

Observe that the assumption of monotone $g(x) / f(x)$ has been dropped; consequently, neither $\Gamma_{0}$ nor $E$ now possesses the required bounding property. They will be replaced by a new outer bound, $\Gamma_{0}^{*}$, (or $v_{0}^{*}$ in the phase plane) and a new inner bound, $E^{*}$, which is the envelope of another family of ovals, $\Omega$.

Furthermore the explicit asymptotic computation of the points near $x=a$ (and $x=b$ ), using Taylor's series expansions, is no longer possible since $f(x)$ and $g(x)$ are not differentiable. It will still be possible, however, to obtain bounds for these coordinates and hence bounds for the amplitude.

5.1 The outer bound modification. Since $-g(x) \leq-g(a)+D(a-x), D$ a positive constant, and $f(x)>K_{1}(a-x)$ in $A-\epsilon \leq x<a$, then the curve

$$
v_{0}^{*}(x)=\frac{-g(a)+D(a-x)}{\mu^{2} K_{1}(a-x)} \geq \frac{-g(x)}{\mu^{2} f(x)}=v_{0}(x), \quad A-\epsilon \leq x<a
$$

lies on, or above, the contour of zero slope in the phase plane. Trajectories on $v_{0}^{*}(x)$ will have negative, or at most, zero slope; thus orbits will move to the right, away from, and below, $v_{0}^{*}(x)$, for the slope of $v_{0}^{*}(x)$ is positive. The contour $v_{0}^{*}(x)$, therefore, is an outer bound in the phase plane and correspondingly, the curve $\Gamma_{0}^{*}$

$$
\Gamma_{0}^{*}: y=F(x)+\frac{-g(a)+D(a-x)}{\mu^{2} K_{1}(a-x)}, \quad A-\epsilon \leq x<a
$$


an outer bound in the Lienard plane.

The point $P_{1}^{*}$ (which replaces $P_{1}$ ) is the point at which the $\chi$ oval of Eq. (3.1) is tangent to $\Gamma_{0}^{*}$.

The equations

$$
\begin{aligned}
& Y_{1}^{*}=F\left(X_{1}^{*}\right)+\frac{-g(a)+D\left(a-X_{1}^{*}\right)}{\mu^{2} K_{1}\left(a-X_{1}^{*}\right)}=F(a)+\left\{2\left[G(k)-G\left(X_{1}^{*}\right)\right]\right\}^{1 / 2} / \mu, \\
& {\left[\frac{d y}{d x}\right]_{X_{1} \cdot Y_{1} \cdot}=\frac{-g\left(X_{1}^{*}\right)}{\mu\left\{2\left[G(k)-G\left(X_{1}^{*}\right)\right]^{1 / 2}\right.}=f\left(X_{1}^{*}\right)+\frac{1}{\mu^{2}} \frac{-g(a)+D\left(a-X_{1}^{*}\right)}{K_{1}\left(a-X_{1}^{*}\right)^{2}}}
\end{aligned}
$$

determine $k_{1}$ and the coordinates $\left(X_{1}^{*}, Y_{1}^{*}\right)$ of $P_{1}^{*}$.

The points $P_{2}, P_{3}, P_{4}$ are determined as in Sec. 2. The point $P_{5}^{*}$ on $\Gamma_{0}^{*}$, replaces $P_{5}$ on $\Gamma_{0}$; the $\operatorname{arc} P_{5}^{*} P_{6}^{*}$, following $\Gamma_{0}^{*}$, replaces the $\operatorname{arc} P_{5} P_{6}$, and the point $P_{6}^{*}$ is determined just as $P_{1}^{*}$ was. The modified outer bound is now complete.

5.2 The inner bound modification. It was remarked, in discussing the bounding properties of the $\chi$ contours, that these contours would be inner bounds as long as the contours remained below $v_{0}(x)$ in the phase plane, or $\Gamma_{0}(x)$ in the Lienard plane. If $v_{0}(x)=-g(x) / \mu^{2} f(x)$ is not monotone increasing, then $v_{0}(x)$ cannot furnish an outer bound on trajectories (hence the use of $v_{0}^{*}(x)$ ), and the justification of the envelope, $E$, as inner bound is no longer valid.

The requirement that $g / f$ be monotone can be circumvented by introducing another set of ovals, $\Omega(x, y)=$ constant, whose envelope, $E^{*}$, still provides an inner bound on trajectories.

The $\Omega$ contours, a family of "shifted" ovals, are defined by

$$
\Omega: \quad \frac{1}{2}[y-F(u)]^{2}+G(x) / \mu^{2}=G(u) / \mu^{2},
$$

where the parameter $u$ is to assume values $u<a$.

We discuss the half-plane $x \leq 0$; similar arguments apply for $x>0$.

Along a solution trajectory one has that

$$
\frac{d \Omega}{d t}=[y-F(u)] \frac{d y}{d t}+g(x) \frac{d x}{d t} / \mu^{2}=-g(x)[F(x)-F(u)] / \mu \geq 0
$$

whenever $F(x) \leq F(u)$ for $x \leq 0$.

Observe that the largest $x$ excursion of an $\Omega$ oval occurs at $x=u$, where $y=F(x)=F(u)$. Furthermore, if $u=\alpha$, then $F(u)=F(\alpha)=0$, and this particular $\Omega$ oval reduces to the contour $\phi_{\alpha}$.

Consider now a trajectory which begins (at some $t=t_{1}$ ) at a point $p_{1}^{*}$ on $y=F(x)$ (see Fig. 10). We assume that $p_{1}^{*}$ is exterior to $\phi_{\alpha}$, as shown. If we choose an $\Omega$ oval which passes through $p_{1}^{*}$ the corresponding value of $u, u_{1}$, will satisfy $u_{1}<\alpha$.

We now follow the trajectory, in time, after this intersection with $y=F(x)$. Observe that the trajectory through $p_{1}^{*}$ will lie exterior not only to the above $\Omega$ oval, but to all $\Omega$ ovals which provide inner bounds for the trajectory. Since all these ovals project somewhat above the curve $y=F(x)$, the trajectory through $p_{1}^{*}$ will lie above the projections, for $x<a$. In particular the trajectory will lie above the envelope to the family (5.2) in $x<a$ (Fig. 11). 


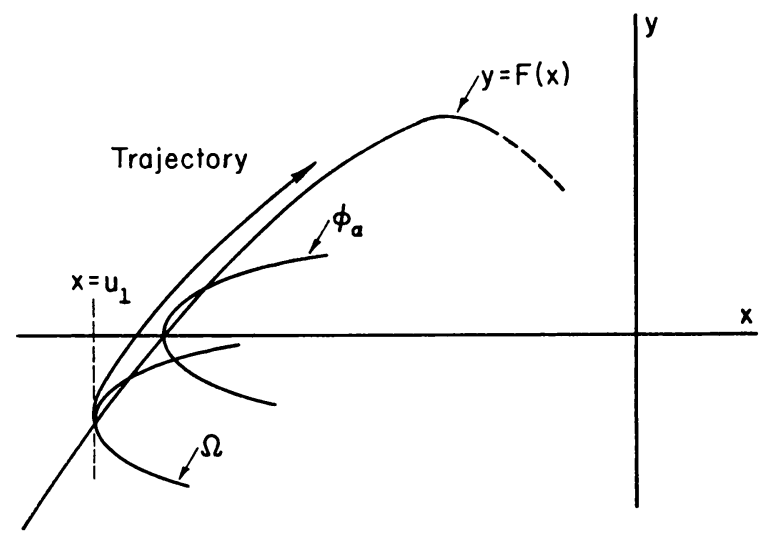

Fig. 10. The excluding property of the $\Omega$ ovals is depicted, in the Liénard plane.

Let the envelope be denoted by $E^{*}$. A parametric representation for $E^{*}$ is

$$
\begin{aligned}
E: \quad y & =F(u)-\frac{g(u)}{\mu^{2} f(u)}, \\
G(x) & =G(u)-\frac{1}{2 \mu^{2}}\left[\frac{g(u)}{f(u)}\right]^{2} .
\end{aligned}
$$

Eqs. (5.3) furnish, for a given value of the parameter $u$, a point, $q_{u}$ on the envelope $E^{*}$. Figure 12 illustrates the geometrical significance of Eqs. (5.3). The point $q_{u}$ is the solution to Eqs. (5.3) and the locus of points $q_{u}$ is the envelope, $E^{*}$. Observe that an excluding interval exists, as before, where $E^{*}$ is an inner bound.

The coordinates of $q_{u_{m}}\left(x_{m}, y_{m}\right)$, the point at the edge of the excluding interval, and the maximum value of the parameter $u, u_{m}$, are solutions of the system

$$
\begin{aligned}
F(x) & =F(u), \\
y & =F(u)-g(u) / \mu^{2} f(u), \\
G(x) & =G(u)-\frac{1}{2 \mu^{2}}[g(u) / f(u)]^{2} .
\end{aligned}
$$

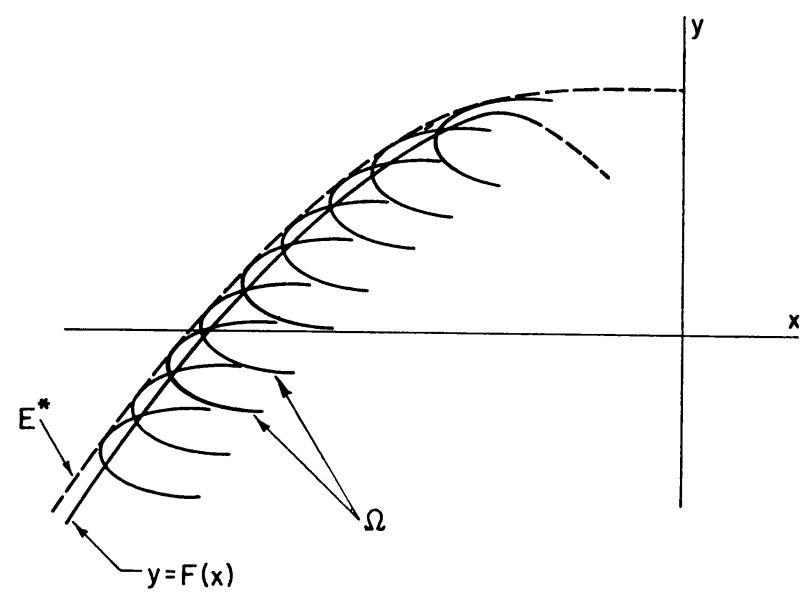

FIg. 11. The envelope, $E^{*}$, of the family of $\Omega$ ovals is shown, in the Liénard plane. 


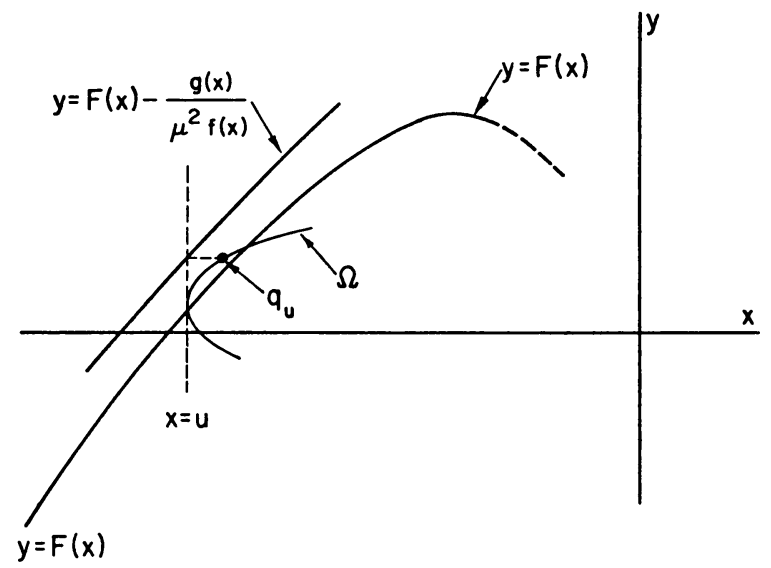

Fig. 12. A geometrical interpretation of the parametric equations for the envelope $E^{*}$ is illustrated, in the Lienard plane.

It is evident that $u_{m}<a<x_{m}$. The point $q_{u_{m}}$ corresponds to the point $p_{u_{m}}=p_{3}$. Indeed, inspection of Eqs. (4.3) reveals that $q_{u_{m}}=p_{u_{m}}$, that is, both $E$ and $E^{*}$ terminate at the same point, $p_{3}$, which is determined from the functions $f, g, F, G$ alone and not from other points on the inner boundary.

The continuation of the inner bound proceeds, as before, from $p_{3}$ through $p_{4}$ to $p_{5}$. From $p_{5}$, however, we continue now with a $\Omega$ oval which intersects $F(x)$ (at $p_{6}^{*}$ ) and joins onto $E^{*}$ at $p_{7}^{*}$. The inner boundary follows $E^{*}$ to $p_{8}^{*}$, where $E^{*}$ ceases to be an inner bound. The points $p_{7}^{*}$ and $p_{2}^{*}$ are in correspondence, as are $p_{8}^{*}$ and $p_{3}^{*}$.

The modified inner bound is complete.

5.3 Nested limit cycles. Figure 13 shows a characteristic curve $y=F(x)$, in the Liénard plane, for which we can apply the results of the previous sections. We assume here that $x g(x)>0$ for all $x$, within the region depicted in the diagram.

We may deduce at once the location of two closed periodic orbits (by generating an annulus for each), namely the limit cycles marked $c_{1}$ and $c_{3}$.

It is shown in the appendix that $c_{1}$ and $c_{3}$ are periodic solutions which possess "orbital stability", that is, all trajectories which begin (at some $t=t$ ) sufficiently near $c_{1}\left(c_{3}\right)$ will converge, as $t \rightarrow \infty$, to $c_{1}\left(c_{3}\right)$. Indeed, the technique described in the preceding sections

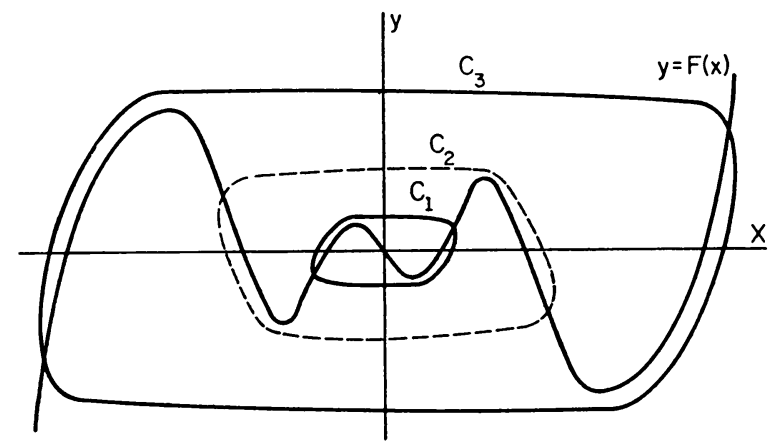

FIg. 13. Nested limit cycles in the Liénard plane; $c_{1}$ and $c_{3}$ are stable, $c_{2}$ is unstable. 
will locate only orbitally stable periodic solutions. However, an unstable non-zero periodic solution exists for the situation shown in Fig. 13. We locate this unstable (i.e. orbitally unstable) solution by generating an annulus for the trajectories in reversed time; that is, we set $t=-\tau$ in the Eq's. (1.4) and replace $y$ by $-Y$ (in order to maintain clockwise motions in the plane), yielding

$$
d x / d \tau=\mu[Y+F(x)], d Y / d \tau=-g(x) / \mu .
$$

Consequently, motions in reversed time are determined by the characteristic curve $Y=-F(x)$, shown in Fig. 14. The limit cycle labelled $c_{2}^{\prime}$ is now evident. It is stable (as $\tau \rightarrow \infty$ ), and an annulus may be constructed for it. Returning again to direct motions, the corresponding solution is unstable (as $t \rightarrow \infty$ ), and is shown dotted in Fig. 13 .

6. Numerical values for the van der Pol equation. For Eq. (1.2), $f(x)=x^{2}-1$, $F(x)=x^{3} / 3-x$ and $g(x)=x$. The zeros of $f(x)$ occur at $a=-1$ and $b=1$, and $F(-1)=-F(1)=\frac{2}{3}$. The constants $A$ and $B$, determined from $F(A)=-\frac{2}{3}$ and $F(B)=\frac{2}{3}$, are $A=-2$ and $B=2$. We also have $F(-1)-F(x)=(2-x)(x+1)^{2} / 3$ so that $L_{1}=\max [(2-x) / 3]=1$ and $L_{2}=\min [(2-x) / 3]=\frac{2}{3}$, in $-1 \leq x \leq 0$. Due to symmetry, $L_{3}=L_{1}$ and $L_{4}=L_{2}$.

We calculate the upper bound on the amplitude of the periodic orbit. Using Eq's. (3.7) to (3.11), we have the quartic $\left(\Delta_{2}^{2}+0.596\right)^{2}=9\left(\Delta_{2}+0.710\right) / 2$ which determines $\Delta_{2}$ (the unique positive root), namely $\Delta_{2}=1.63$. From Eq. (3.12) we obtain the upper bound as $2+0.857 \mu^{-4 / 3}+O\left(\mu^{-2}\right)$.

The lower bound, from Eq. (4.8), is found to be $2+0.643 \mu^{-4 / 3}+O\left(\log \mu / \mu^{2}\right)$.

These bounds are to be compared with the exact asymptotic expansion of the amplitude, which is $2+0.779 \mu^{-4 / 3}+O\left(\log \mu / \mu^{2}\right),[6]$.

If we treat the van der Pol equation according to the scheme outlined in Section 5, we have $f(x)=x^{2}-1>2(-1-x)$ in $x<-1$, so that $K_{1}=2$. Noting that $K_{1}=\left|f^{\prime}(-1)\right|$, so that $\Gamma_{0}^{*}$ agrees, to within $O\left(\mu^{-2}\right)$, with $\Gamma_{0}$ at $P_{1}$, we find that the upper bound on the amplitude does not change from that given above. For the lower bound, observe that $p_{3}$, where the envelope $E^{*}$ was terminated, is identical with the point at which the envelope $E$ was terminated. Consequently, the lower bound will agree exactly with that given above. Indeed, if $f(x)>\left|f^{\prime}(a)\right|(a-x)$ in $A-\epsilon \leq x<a$, so that $K_{1}=\left|f^{\prime}(a)\right|$, then the amplitude bounds determined from Section 5 will agree, to within $O\left(\mu^{-2}\right)$, with those computed from Sections 3 and 4, with $\left|f^{\prime}(a)\right|$ replaced by $K_{1}$.

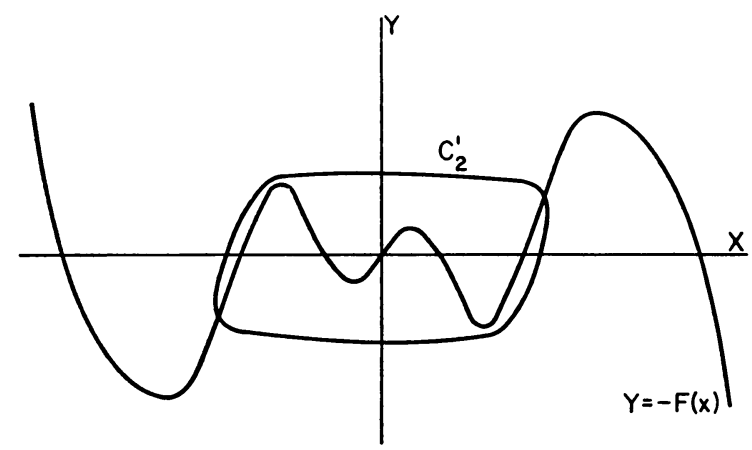

Fig. 14. The unstable limit cycle, $c_{2}$ of figure 13, is represented in reversed time, where it becomes a stable periodic orbit, $c_{2}^{\prime}$. 


\section{APPENDIX: Uniqueness of the Periodic Orbit Within an Annulus}

We consider periodic solutions to the generalized Liénard equation, $d^{2} x / d t^{2}+$ $f(x) d x / d t+g(x)=0$, in the Lienard plane defined by $d x / d t=y-F(x), d y / d t=$ $-g(x)$ where $F(x)=\int_{0}^{x} f(\xi) d \xi$. Note that we do not introduce a parameter $\mu$, since our proof is parameter independent. We suppose, however, that $f(x), F(x)$ and $g(x)$ satisfy the conditions of Section 5 and that a confining annulus has been obtained.

Trajectories which begin (at $t=0$ say) on the positive $y$-axis will return to the positive $y$-axis after one revolution about the origin. This defines a continuous transformation of the positive $y$-axis onto itself. Fixed points of this transformation identify periodic orbits. To demonstrate uniqueness, we show that there can be no more than one fixed point on that portion of the positive $y$-axis which lies within the annulus; that is, at most one periodic orbit can exist within the annulus. Since the PoincareBendixson theorem guarantees at least one periodic orbit within a confining annulus, we can conclude that it is unique. We now proceed to the investigation of the transformation.

Figure 15 shows two arbitrary trajectories, $y_{1}(x)$ and $y_{2}(x)$, within the annulus. Let the inner trajectory, $y_{1}(x)$, intersect $y=F(x)$ at $x=x_{0}$, and set $M=F\left(x_{0}\right)$. It is clear from the conditions on $F(x)$ that (i) $F(x) \leq M$ in $0 \leq x \leq x_{0}$ and (ii) $F(x)>M>0$ in that portion of the annulus for which $x>x_{0}$.

Consider the function $\chi(x, y)=(y-M)^{2} / 2+G(x)$. We have, along a solution trajectory, that

$$
d x=(y-M) d y+g(x) d x=[F(x)-M] d y=\frac{g(x)[M-F(x)] d x}{y-F(x)} .
$$

Along $y_{1}(x)$ and $y_{2}(x)$,

$$
\chi(H)-\chi(A)=\int_{A}^{H} d \chi=\int_{0}^{x_{0}} g(x)[M-F(x)] d x /\left[y_{1}-F(x)\right],
$$

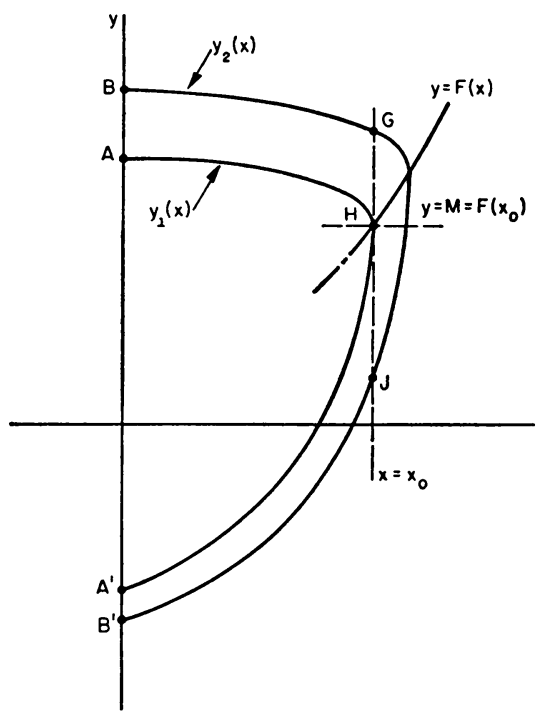

FIG. 15. Two trajectories within a confining annulus, in the Lienard plane. 
and

respectively.

$$
\chi(G)-\chi(B)=\int_{B}^{G} d \chi=\int_{0}^{x_{0}} g(x)[M-F(x)] d x /\left[y_{2}-F(x)\right],
$$

Since $y_{2}-F(x)>y_{1}-F(x)$, over the $\operatorname{arcs} A H$ and $B G$ in $\left(O, x_{0}\right)$, then

$$
\chi(G)-\chi(B)<\chi(H)-\chi(A) .
$$

One also has, along $y_{2}(x)$, that

$$
\chi(J)-\chi(G)=\int_{G}^{J}[F(x)-M] d y<0,
$$

since $F(x)>M$ and $d y<0$.

Finally, as with the first inequality, we obtain

$$
\chi\left(B^{\prime}\right)-\chi(J)<\chi\left(A^{\prime}\right)-\chi(H) .
$$

Adding the three inequalities, we have

$$
\chi\left(B^{\prime}\right)-\chi(B)<\chi\left(A^{\prime}\right)-\chi(A) .
$$

Substituting the expression for $\chi(x, y)$, and rearranging somewhat, yields

$$
y^{2}\left(B^{\prime}\right)-y^{2}\left(A^{\prime}\right)<y^{2}(B)-y^{2}(A)-2 M\left[y(B)-y(A)+y\left(A^{\prime}\right)-y\left(B^{\prime}\right)\right]
$$

so that

$$
y^{2}\left(B^{\prime}\right)-y^{2}\left(A^{\prime}\right)<y^{2}(B)-y^{2}(A) .
$$

In exactly the same way, we continue the trajectories into $x \leq 0$ and show that $y^{2}\left(B^{\prime \prime}\right)-y^{2}\left(A^{\prime \prime}\right)<y^{2}\left(B^{\prime}\right)-y^{2}\left(A^{\prime}\right)$, where $A^{\prime \prime}$ and $B^{\prime \prime}$ are the points on the positive $y$-axis to which the $A$ and $B$ trajectories (respectively) return, after making one revolution. Consequently, we have

$$
y^{2}\left(B^{\prime \prime}\right)-y^{2}\left(A^{\prime \prime}\right)<y^{2}(B)-y^{2}(A) .
$$

Note that this is a strict inequality.

Assume now that at least two fixed points of the transformation exist (implying the existence of two or more periodic orbits within the annulus). On letting $A$ and $B$ be two such fixed points we have $y\left(A^{\prime \prime}\right)=y(A), y\left(B^{\prime \prime}\right)=y(B)$, contradicting the strict inequality. Hence there is at most, therefore exactly, one fixed point within the annulus. The existence of a unique periodic orbit within such a confining annulus is thus established. Note that the mapping is contractional, so that these periodic solutions are orbitally stable.

\section{BIBLIOGRAPHY}

1. L. Cesari, Asymptotic behaviour and stability problems in ordinary differential equations, Ergebnisse der Mathematik, New Series, vol. 16, Academic Press Inc., New York, 1963 (2nd edition)

2. J. LaSalle, Relaxation oscillations, Q. Appl. Math. 7 (1949) 1-19

3. N. Levinson and O. K. Smith, $A$ general equation for relaxation oscillations, Duke Math. Journal 9 (1942) 382-403

4. J. LaSalle and S. Lefschetz, Stability by Liapunov's direct method, with applications, Academic Press, New York, 1961, p. 62

5. M. L. Cartwright, Forced oscillations in nonlinear systems, in Annals of Mathematics Studies, No. 20, edited by S. Lefschetz, Princeton University Press, Princeton, 1950, pp. 152-153

6. A. A. Dorodnytsin, Asymptotic solution of van der Pol's equation, Prikl. Mat. Mech. 11 (1947) pp. 313-328 Am. Math. Soc. Transl. no. 88 